\title{
A novel variable delay Go/No-Go task to study attention, motivation and working memory in the head-fixed rodent
}

\section{[version 1; peer review: 1 approved, 1 approved with}

\section{reservations]}

\author{
Samuel D Dolzani1,2, Shinya Nakamura1, Donald C Cooper ${ }^{1,2}$ \\ ${ }^{1}$ Institute for Behavioral Genetics, University of Colorado, Boulder, CO, 80309, USA \\ 2Department of Psychology and Neuroscience, University of Colorado, Boulder, CO, 80303, USA
}

V1 First published: 10 May 2013, 2:125

https://doi.org/10.12688/f1000research.2-125.v1

Latest published: 19 Mar 2014, 2:125

https://doi.org/10.12688/f1000research.2-125.v2

\begin{abstract}
In order to parse the causal elements underlying complex behaviors and decision-making processes, appropriate behavioral methods must be developed and used in concurrence with molecular, pharmacological, and electrophysiological approaches. Presented is a protocol for a novel Go/No-Go behavioral paradigm to study the brain attention and motivation/reward circuitry in awake, head-restrained rodents. This experimental setup allows: (1) Pharmacological and viral manipulation of various brain regions via targeted guide cannula; (2) Optogenetic cell-type specific activation and silencing with simultaneous electrophysiological recording and; (3) Repeated electrophysiological single and multiple unit recordings during ongoing behavior. The task consists of three components. The subject first makes an observing response by initiating a trial by lever pressing in response to distinctive Go or No-Go tones. Then, after a variable delay period, the subject is presented with a challenge period cued by white noise during which they must respond with a lever press for the Go condition or withhold from lever pressing for the duration of the cue in the No-Go condition. After correctly responding during the challenge period (Challenge) and a brief delay, a final reward tone of the same frequency as the initiation tone is presented and sucrose reward delivery is available and contingent upon lever pressing. Here, we provide a novel procedure and validating data set that allows researchers to study and manipulate components of behavior such as attention, motivation, impulsivity, and rewardrelated working memory during an ongoing operant behavioral task while limiting interference from non task-related behaviors.
\end{abstract}

Keywords

Go/No-Go, attention, motivation, working memory, head-fixed rodent

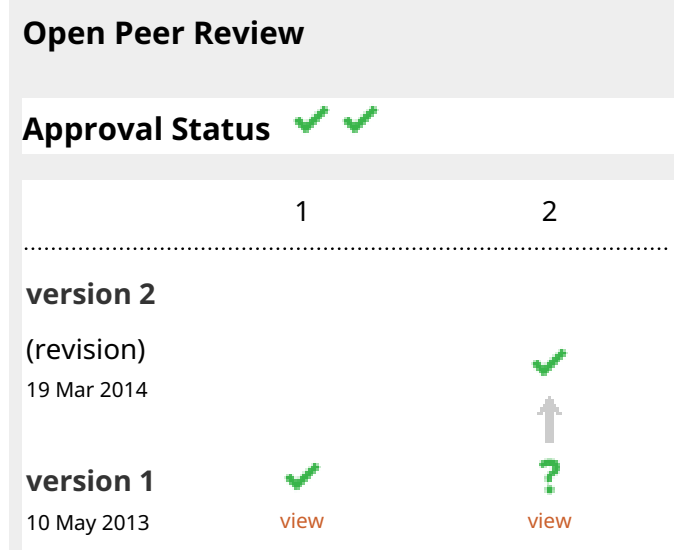

1. Kenichiro Tsutsui, Tohoku University, Sendai, Japan

2. Jeff Dalley, University of Cambridge, Cambridge, UK

Any reports and responses or comments on the article can be found at the end of the article. 
Corresponding author: Donald C Cooper (d.cooper@colorado.edu)

Competing interests: No competing interests were disclosed.

Grant information: This work was supported by R01 DA24040 (DCC).

The funders had no role in study design, data collection and analysis, decision to publish, or preparation of the manuscript.

Copyright: (c) 2013 Dolzani SD et al. This is an open access article distributed under the terms of the Creative Commons Attribution

License, which permits unrestricted use, distribution, and reproduction in any medium, provided the original work is properly cited. Data associated with the article are available under the terms of the Creative Commons Zero "No rights reserved" data waiver (CC0 1.0 Public domain dedication).

How to cite this article: Dolzani SD, Nakamura S and Cooper DC. A novel variable delay Go/No-Go task to study attention, motivation and working memory in the head-fixed rodent [version 1; peer review: 1 approved, 1 approved with reservations] F1000Research 2013, 2:125 https://doi.org/10.12688/f1000research.2-125.v1

First published: 10 May 2013, 2:125 https://doi.org/10.12688/f1000research.2-125.v1 


\section{Introduction}

The use of operant behavioral tasks that utilize the headimmobilized condition in rodents have some advantages over tasks that occur under freely moving conditions by limiting the range of possible non task-relevant behaviors and distractors. While the head-restrained behaving rodent has been examined for many years, the relevant literature is minimal in comparison to similar behavioral tasks involving freely moving animals. Head immobilization is common in acute electrophysiological recordings and has been extended to study cardiovascular function, neuroimaging, reflex adaptation, and licking movements ${ }^{1}$. To date, very few experiments have assessed psychological and physiological processes simultaneously occurring during operant behavioral tasks in the awake, head-restrained rodent ${ }^{1}$.

The Go/No-Go task, a canonical paradigm in animal behavior and psychology, requires the subject to initiate an operant conditioned response during one stimulus $\left(\mathrm{CS}_{+}\right)$and withhold from such a response during the presence of the opposite type of stimulus (CS-) ${ }^{1,2}$. This type of behavioral task has a high level of construct validity and clinical utility when studying the brain motivation/reward pathways and behaviors such as impulsivity. Impulsivity and motivational deficits are complex behavioral phenotypes implicated in a number of psychiatric disorders including attention deficit hyperactivity disorder (ADHD), mania and depression associated with bipolar disorder, schizophrenia, pathological gambling, borderline personality disorder, and substance abuse $e^{2}$. Utilization of an appropriate variant of the rodent Go/No-Go task allows for these complex, clinically relevant behaviors to be interrogated and manipulated. Data obtained from these studies provide the opportunity for development of novel future pharmacotherapies and behavioral interventions ${ }^{2}$.

The head-immobilized rodent Go/No-Go task we have developed is a procedure that provides the framework for performing repeated electrophysiological recordings and simultaneous molecular and pharmacologic interventions during ongoing operant behavioral tasks. In addition, this technique allows for accurate stereotaxic localization during electrophysiological recordings, daily anesthesia-free recordings in various brain regions, rapid electrode repositioning during chronic recording sessions, and high quality stabilized recordings ${ }^{3}$. With the advent of optogenetics, the head-restrained procedure allows for these recordings to be coupled with cell-type specific silencing and activation, free from electrophysiological artifacts ${ }^{4,5}$.

\section{Methods}

Animals

Four male Sprague Dawley rats and 28 male Long Evans rats (8 weeks and 200-300 g at time of surgery), bred at the University of Colorado Boulder, were used for all experiments. Animals were maintained on a $12 \mathrm{~h}$ reverse light/dark cycle (lights off at 07:00). The behavioral experiments were conducted during the dark period. Animals were singly housed and water-restricted, while food was available ad libitum for the duration of the experiment. All procedures were in compliance with animal care standards set forth by the Institute for Animal Care and Use Committee at the University of Colorado Boulder.

\section{Surgical preparation}

Animals were stereotaxically affixed with stainless steel headcaps prior to any behavioral training. Rats were deeply anesthetized with an intraperitoneal injection of ketamine-xylazine (Sigma-Aldrich; $80 \mathrm{mg} / \mathrm{ml} \mathrm{ketamine/6} \mathrm{mg/ml} \mathrm{xylazine)} \mathrm{and}$ restrained on a custom stereotaxic device (Old School Industries, CO, USA). Head fur was shaved and a rostro-caudal incision was made from the region roughly $10 \mathrm{~mm}$ anterior to bregma, extending 5-7 $\mathrm{mm}$ posterior to lamba. Connective tissue on the skull was carefully removed and temporal muscles were retracted from the dorsolateral region of the skull. Vasculature was cauterized using 30\% hydrogen peroxide (SigmaAldrich, USA) and quickly rinsed with sterilized saline. Eight self-tapping screws (Small Parts, IN, USA) were implanted on the dorsal surface of the skull and four screws were implanted on the lateral region of the skull to confer head-cap stability and strength. After screws were implanted, the skull was sterilized using povidone-iodine (9\%) (Sigma-Aldrich, USA) then desiccated with sterile cotton swabs. A thin layer of radiopaque glass ionomer restorative cement (GC America Inc., USA) was applied to the skull and around the base of the reinforcing screws to increase adhesion strength between the skull and head holder cap. After allowing the radiopaque cement to set, two horizontal stainless steel head restraint bars were stereotaxically positioned directly above the exposed dorsal region of the skull ( $5 \mathrm{~mm}$ anterior to bregma and $3 \mathrm{~mm}$ posterior to lamda). Bars were positioned approximately $5 \mathrm{~mm}$ above the dorsal surface of the skull and acrylic dental cement (Stoelting, USA) was applied between the head restraint bars and the skull. After implantation of screws and head restraint bars, surrounding areas of the skull were disinfected using povidone-iodine and antibiotic ointment (Sigma-Aldrich, USA) was applied around the site of incision. Animals were given 7 days to recover with food and water available ad libitum.

\section{Experimental setup}

All behavioral training was conducted using a custom headrestraint system (Old School Industries, CO, USA) in a red acrylic box. A reward-delivery nozzle, lever, sensor systems for detecting licking movements and lever-presses, and a speaker were placed around the head-restraint stage. All computer software used for the experiments was customized using LabVIEW (National Instruments, USA) and a digital input/output interface (USB-6008, National Instruments, USA) was used for controlling all devices. 


\section{Behavioral conditioning procedure}

Habituation to training device

After animals recovered from surgery, they were handled for $3-5$ days and allowed to explore $(20 \mathrm{~min} /$ day $)$ the operant conditioning boxes that they were restrained within for the remaining duration of the experiment. The purpose of this was to familiarize the animal with the experimental environment and reduce context-related stress and excitability. Subjects were head restrained on an ergonomically designed custom head-restraint system (Old School Industries, CO, USA) for 60 minutes/day during the habituation period of the training procedure. During this time period, subjects were administered $0.3 \mathrm{M}$ sucrose (Sigma-Aldrich, USA) non-contingently through a reward delivery nozzle positioned near the mouth. Approximately $40 \mu \mathrm{l}$ sucrose was delivered every 10 seconds (10 s inter-trial interval (ITI)) over the course of the 60 minute habituation session, yielding a total reward delivery of $15 \mathrm{ml} / 60$ minute session.
Acquisition of lever-pressing and lever/tone association Following successful sucrose consumption under non-contingent reward delivery conditions, subjects were trained to press a right forepaw lever to receive sucrose reinforcement (Figure 1). This stage of operant behavioral training was intended to facilitate the contingent relationship between lever pressing and reward delivery. This phase of training persisted until the subject responded with $>1000$ lever presses for 2-3 consecutive days. days. The next phase of training was the Lever/Tone association phase during which subjects learned to lever press in the presence of an audible tone. The two tones randomly presented were either $3000 \mathrm{~Hz}$ or $9000 \mathrm{~Hz}$, which corresponded to the same frequencies used in the Go and No-Go constituents of the later stages of the training procedure. This stage of training began with a $5 \mathrm{~s}$ tone presentation with $8 \mu \mathrm{l}$ sucrose delivered for every lever press occurring during the tone (15 s ITI). Subjects were switched to the next stage of training after $>70 \%$ correct

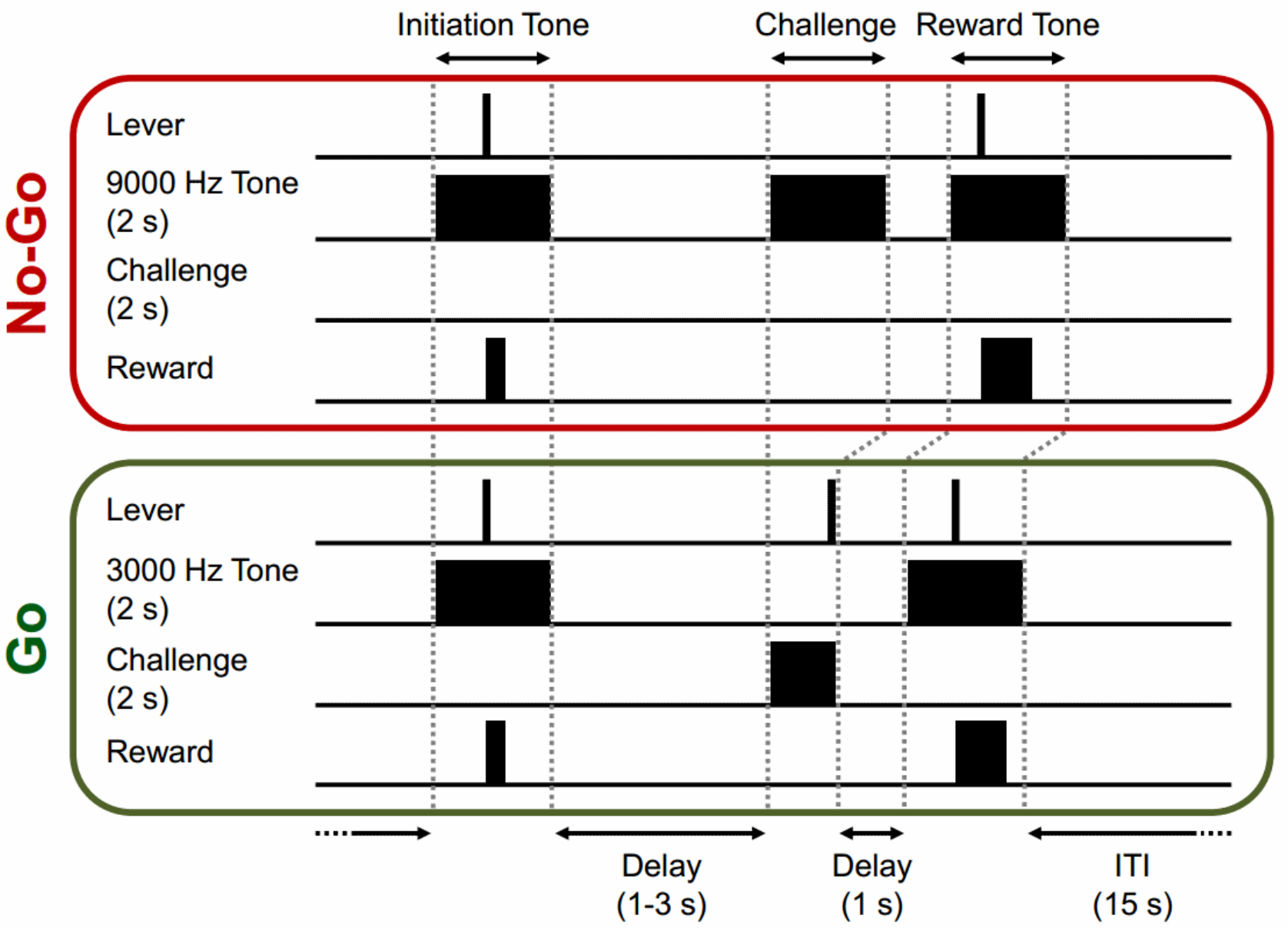

Figure 1 Schematic showing the head-fixed Go/No Go task under a No-Go trial and Go trial. Lever presses, initiation tone, challenge period, reward tone, and sucrose reward delivery components are shown for both trials. The black boxes represent the relative timing of the rats response (lever press) in response to the auditory cues. ITI: inter-trial interval. 
responding during the presence of the tone. The next phase of training was a reduction in the duration of the tone presentation ( $5 \mathrm{~s}$ to $3 \mathrm{~s}$ ). After achieving $>70 \%$ correct responding using these parameters, tone duration was finally decreased to $2 s$ with a single lever press contingent sucrose delivery of $40 \mu \mathrm{l}$. The final phase persisted until subjects achieved $>80 \%$ correct responding for 2 consecutive sessions.

\section{No-Go acquisition}

First, subjects were trained to perform the No-Go constituent of the behavioral task after successfully completing the Lever/ Tone association phase (Figure 1). During No-Go trials, the subject was required to initiate a trial by lever pressing in response to $3000-\mathrm{Hz}, 2-\mathrm{s}$ tone (initiation tone). Correct trial initiation yielded an $8 \mu \mathrm{l}$ sucrose delivery. Following the initiation tone response, a variable delay (1-3 s) was followed by a 2-s white-noise presentation (challenge period). This was the specific epoch in which the subject must withhold from lever pressing (No-Go). Following successful suppression of lever pressing during the challenge period, the $2-\mathrm{s} 3000-\mathrm{Hz}$ tone was presented again (reward tone). A second lever press was required during the presence of the final reward tone and a larger $40 \mu \mathrm{l}$ sucrose was delivered in response to this lever press. Following a 15-s ITI, the next initiation tone was presented. If the subject failed to initiate a trial or withhold from lever pressing during the challenge period, the trial was terminated and a 15-s ITI followed. No-Go acquisition training proceeded until the subject responded at $>80 \%$ correct challenge period responding for two consecutive days before moving to the Go condition training.

\section{Go acquisition.}

After successful acquisition of the No-Go constituent of the training procedure, subjects began daily 90 minute Go training sessions (Figure 1). In a Go trial, subjects must lever press to initiate a trial during the presence of a $9000-\mathrm{Hz}, 2-\mathrm{s}$ initiation tone. $8 \mu \mathrm{l}$ of sucrose was delivered in response to the lever press. Following initiation of a trial, a 2 -s white noise (challenge period) was presented after a variable delay (1-3 s). The subject must lever press (Go) during the challenge period. Lever pressing during the challenge period terminated the white noise and was followed by a 2-s final reward tone $(9000 \mathrm{~Hz})$. The larger $40 \mu \mathrm{l}$ sucrose reward was only delivered if the subject correctly lever pressed during the reward tone. In the early stage of Go training, a white noise signal of indefinite duration was used for the challenge period. As subjects learned the association between the $9000-\mathrm{Hz}$ initiation tone and lever pressing during the challenge period their reaction time decreased and the challenge period duration was decreased to $2 \mathrm{~s}$. Subjects were trained using these parameters until they achieved $>80 \%$ correct responding during the 2-s challenge period for 2 consecutive days before a randomized within session Go/No Go condition began (data not included).

\section{Results}

Subjects rapidly acquired the ability to consume sucrose delivered non-contingently through a reward delivery nozzle. Following acquisition of sucrose consummatory behavior, subjects quickly learned ( $<3$ days) an operant response by lever pressing (Lever) to receive sucrose reinforcement (Figure 2). On average, subjects reached the specified criteria of $>1000$ presses $/ 90$ minute training session in $<3$ consecutive daily training sessions $(n=24)$. Next, subjects reliably acquired operant lever pressing behavior during the presence of a tone (Lever/Tone association) to receive sucrose reinforcement (Figure 2). The Lever/Tone association stage of training required an average of 8 training sessions to achieve the criteria of $>70 \%$ correct responding for two consecutive days $(n=20)$. This requisite stage of training was intended to facilitate the association between tone presentation, lever pressing, and concomitant primary reinforcement. No-Go training (No-Go) followed the Lever/Tone association training (Figure 2). The NoGo constituent of the behavioral task required an average of 22 daily training sessions to achieve criteria of $>80 \%$ correct challenge period responding $(n=14)$. Finally, subjects were trained to perform the Go constituent (Go) of the Go/No-Go task (Figure 2). This component of the task required an average of 14 daily training sessions to achieve a criteria of $>80 \%$ correct $2 \mathrm{~s}$ challenge period responding for 2 consecutive days $(\mathrm{n}=13)$. Raster plots of five consecutive representative No-Go trials (Figure 3A) and histograms of lever pressing and licking during the initiation tone, challenge period, and reward tone are shown (Figure 3B). The histograms represent cumulative responding across a 90 minute training session. Subjects lever pressed in response to an initiation tone and received an initial small sucrose reinforcement $(8 \mu \mathrm{l})$, withheld from lever pressing (No-Go) during the challenge period, and emitted a final lever press during the final reward tone to receive a larger $40 \mu \mathrm{l}$ sucrose reinforcement. One

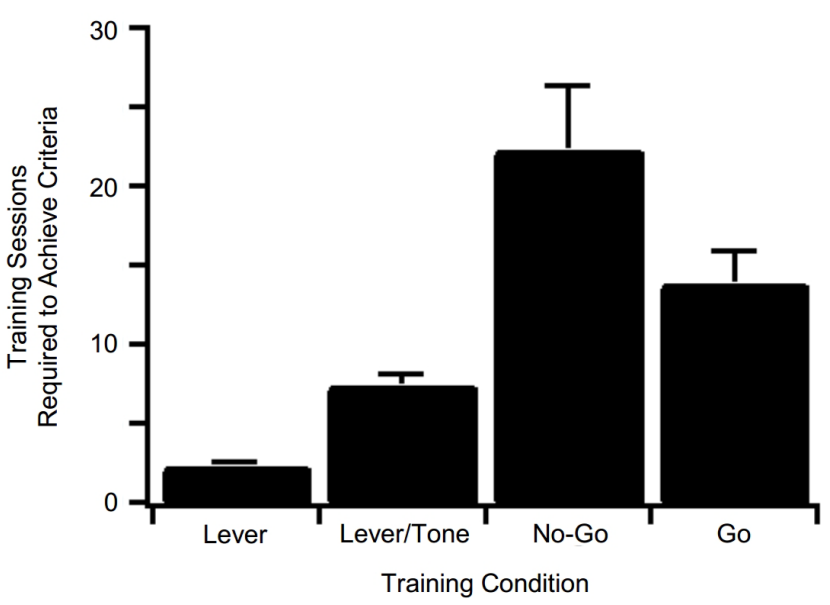

Figure 2 The average and standard error of the mean training sessions required to achieve specified criteria for acquisition of each head-fixed training condition of the behavioral task is shown. Lever: $n=24$, Lever/Tone: $n=20$, No-Go: $n=14$, Go: $n=13$. 
A.

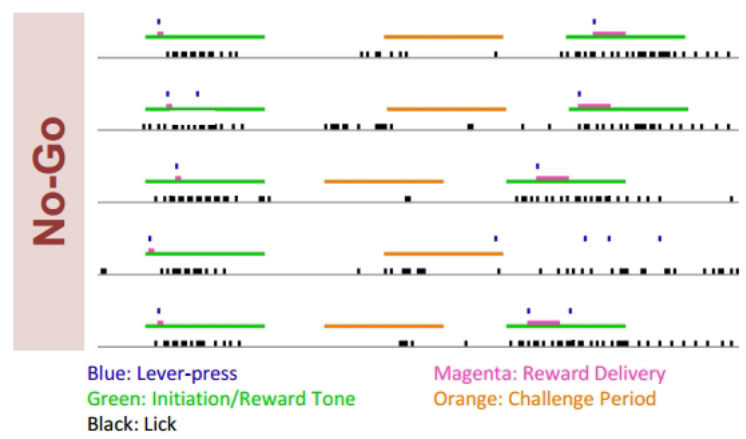

B.

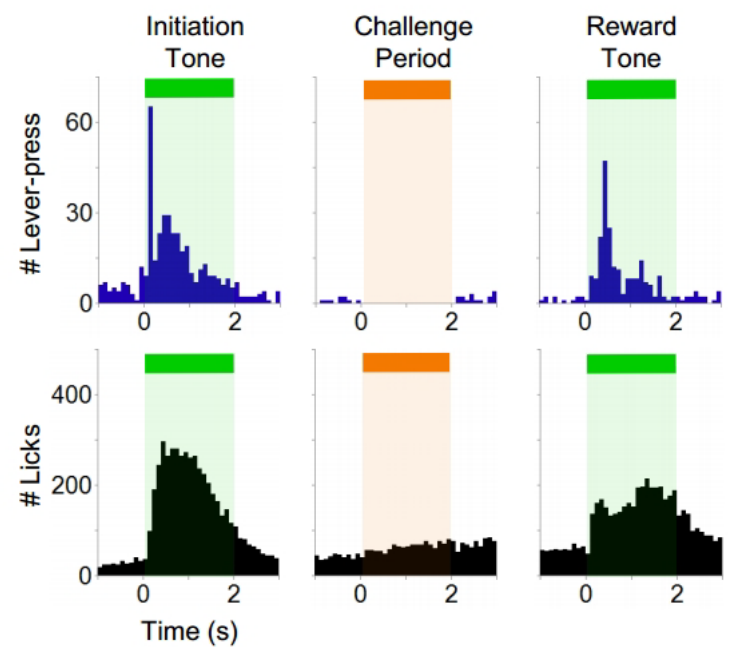

Figure 3 Raster plot of five consecutive No-Go trials (A) and histograms of lever pressing and licking during initiation tone, challenge period, and reward tone (B). Green bar: initiation tone and reward tone, orange bar: challenge period, magenta bar: reward delivery, black: lick, blue: lever press. (Histogram bin size $=100 \mathrm{~ms}$ )

example of a failed No-Go trial is shown ( $4^{\text {th }}$ trial on the raster plot). The subject emitted a lever press during the challenge period of the No-Go trial, resulting in a failed trial and no final $40 \mu$ sucrose delivery. Raster plots of five consecutive Go trials (Figure 4A) and histograms of lever pressing and licking during the initiation tone, challenge period, and reward tone are shown (Figure 4B). Subjects initiated a trial by lever pressing during the initiation tone, emitted a lever press during the challenge period (Go), and lever pressed during the final reward tone to receive sucrose reinforcement. One example of a failed Go trial is shown ( $1^{\text {st }}$ trial on the raster plot). The subject failed to lever press during the challenge period of the Go trial and the final $40 \mu$ sucrose reward was not delivered. The data set from all rats is presented.

Data for 'Go', 'Lever press acquisition', 'Lever tone acquisition' and 'No-go' behavioral tasks

4 Data Files

http://dx.doi.org/10.6084/m9.figshare.654019
A.

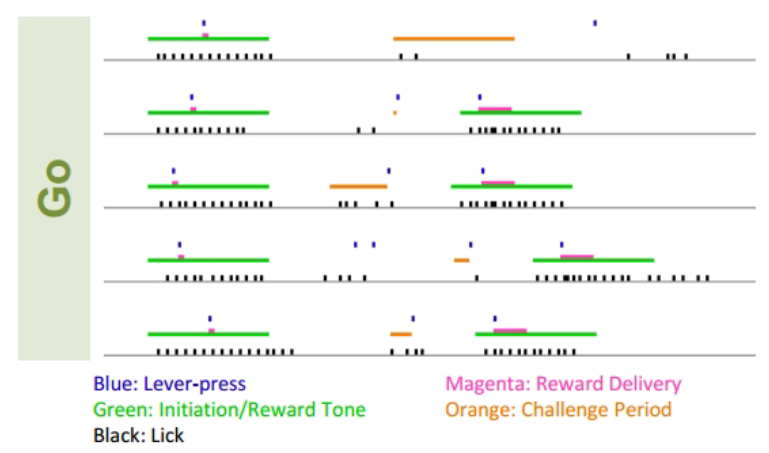

B.

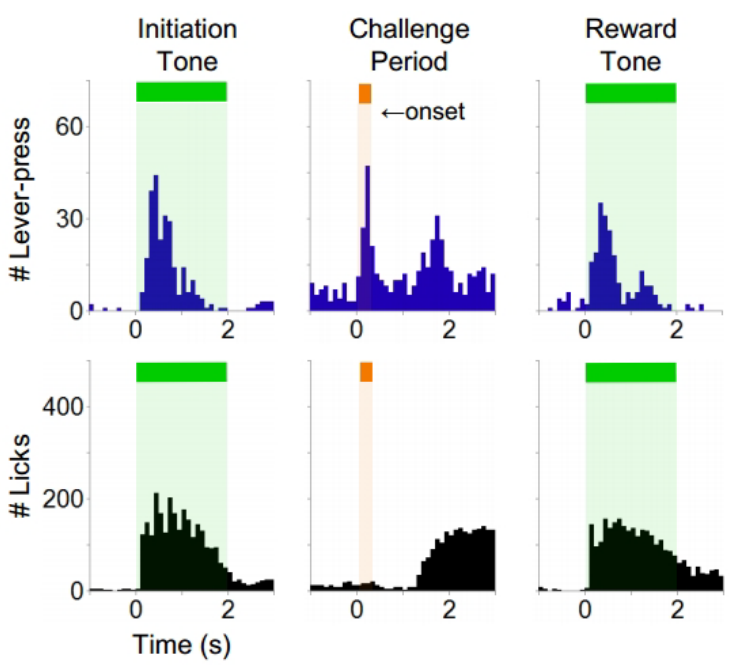

Figure 4 Raster plot of five consecutive Go trials (A) and histograms of lever pressing and licking during the initiation tone, challenge period, and reward tone (B). Green bar: initiation tone and reward tone, orange bar: challenge period, magenta bar: reward delivery, black: lick, blue: lever press. (Histogram bin size $=100 \mathrm{~ms}$ ). The second peak on the histogram of lever pressing during the challenge period represents lever presses during the final reward tone. Note the emergence of non cued lever presses and licking during the challenge period that represents the idiosyncratic variable initiation during the Go challenge tone and subsequent reward tone delivery under the Go condition.

\section{Author contributions}

SDD, SN, and DCC designed experiments and analyzed the data. SDD and SN performed the experiments. SDD wrote the paper. All authors agreed the final manuscript for publication.

\section{Competing interests}

No competing interests were declared.

Grant information

This work was supported by R01 DA24040 (DCC).

\section{Acknowledgments}

The authors would like to thank Dr. Mike Baratta and Matthew Pomrenze for their insightful commentary and intellectual faculty throughout the duration of the project. 
1. Schwarz C, Hentschke H, Butovas S, et al: The head-fixed behaving rat - Procedures and pitfalls. Somatosens Mot Res. (2010); 27: 131-148.

2. Winstanley CA: The utility of rat models of impulsivity in developing pharmacotherapies for impulsive control disorders. $\mathrm{Br} \mathrm{J}$ Pharmacol. (2011); 164: 1301-21.

3. Chaniary KD, Baron MS, Robinson P, et al: A novel stereotaxic apparatus for neuronal recordings in awake head-restrained rats J Neurosci Methods. (2011); 198: 29-35.

4. Zhang F, Wang LP, Brauner M, et al: Multimodal fast optical interrogation of neural circuitry. Nature. (2007); 446: 633-9.

5. Boyden ES, Zhang F, Bamberg E, et al: Millisecond-timescale, genetically targeted optic control over neural activity. Nat Neurosci. (2005); 8: 1263-8. 


\title{
Open Peer Review
}

\section{Current Peer Review Status:}

\section{Version 1}

Reviewer Report 09 July 2013

https://doi.org/10.5256/f1000research.1165.r1050

(C) 2013 Dalley J. This is an open access peer review report distributed under the terms of the Creative Commons Attribution License, which permits unrestricted use, distribution, and reproduction in any medium, provided the original work is properly cited.

\author{
Jeff Dalley \\ ICCAM Cambridge, Department of Psychiatry, University of Cambridge, Cambridge, UK
}

This is an interesting and well-conducted study on the development and validation of a novel, symmetrically-reinforced Go/No-Go task in rats. The novelty of this procedure presumably rests with head fixing the animals to limit the impact of extraneous behaviour, and the configuration of the task itself, which enables several component cognitive and behavioural processes to be independently assessed. One could imagine using such a task in a variety of settings to assess attention (e.g. based on Posner's task), inhibitory response control, and working memory. However, this is not a radically new approach; for example, head restraint is often used to avoid the use of a general anaesthetic agent in MR and PET imaging and head restraint has been used for decades to facilitate neurophysiological experiments in non-human primates. Furthermore, restraint itself is a potent activator of the stress response which may have unintended consequences for brain-behaviour relationships. It would have been interesting to compare blood corticosterone in head-fixed and non-head-fixed animals. The reported behavioural findings suggest that sucrose was sufficient as a reinforcer but crucially, how stable was performance both within and between sessions?

Overall, the article is clear and concise with methods that are fully transparent. Although the Title and Abstract reflect the content of the article it would be helpful to explain the novel aspects of the present protocol.

Competing Interests: No competing interests were disclosed.

I confirm that I have read this submission and believe that I have an appropriate level of expertise to confirm that it is of an acceptable scientific standard, however I have significant reservations, as outlined above.

Reviewer Report 03 July 2013

https://doi.org/10.5256/f1000research.1165.r1038 
(C) 2013 Tsutsui K. This is an open access peer review report distributed under the terms of the Creative Commons Attribution License, which permits unrestricted use, distribution, and reproduction in any medium, provided the original work is properly cited.

\section{Kenichiro Tsutsui}

Department of Biology, Tohoku University, Sendai, Japan

This interesting paper describes the details of a go/no-go task designed for head-restrained rats. A detailed description about the training procedure is obviously beneficial for the biological community since the use of behaving rats is emerging in optogenetics and electrophysiology studies. I would like to ask the authors to consider the following points for the improvement of the paper. (All are "minor" comments.)

1. Go/no-go tasks have long been commonly used in behavioral psychology, and its application to behavioral electrophysiology with head-restrained monkeys is as early as in 1980s. The authors could briefly mention the history of go/no-go task in the 'Introduction' section.

2. I am not entirely sure which part of the described go/no-go task is "novel". Please point it out clearly in the manuscript which part of this protocol is different from the conventional go/no-go task procedure.

3. How stable is the performance of a typical subject that has reached the criteria of training? It would be helpful to show the weekly or monthly performance of a representative subject after the completion of training.

Competing Interests: No competing interests were disclosed.

I confirm that I have read this submission and believe that I have an appropriate level of expertise to confirm that it is of an acceptable scientific standard. 
The benefits of publishing with F1000Research:

- Your article is published within days, with no editorial bias

- You can publish traditional articles, null/negative results, case reports, data notes and more

- The peer review process is transparent and collaborative

- Your article is indexed in PubMed after passing peer review

- Dedicated customer support at every stage

For pre-submission enquiries, contact research@f1000.com 